\title{
Poétiques du descriptif dans le roman français du XIX siècle, A. de Georges-Métral (dir.)
}

\section{Angela Di Benedetto}

\section{(2) OpenEdition}

1 Journals

\section{Edizione digitale}

URL: http://journals.openedition.org/studifrancesi/9981

DOI: 10.4000/studifrancesi.9981

ISSN: 2421-5856

\section{Editore}

Rosenberg \& Sellier

\section{Edizione cartacea}

Data di pubblicazione: 1 août 2017

Paginazione: 367-368

ISSN: 0039-2944

\section{Notizia bibliografica digitale}

Angela Di Benedetto, «Poétiques du descriptif dans le roman français du XIXe siècle, A. de Georges-Métral (dir.)», Studi Francesi [Online], 182 (LXI | II) | 2017, online dal 01 août 2017, consultato il 11 janvier 2021. URL: http://journals.openedition.org/studifrancesi/9981; DOI: https://doi.org/10.4000/studifrancesi. 9981

Questo documento è stato generato automaticamente il 11 janvier 2021.

\section{(c) $($ ) $\odot$ EY}

Studi Francesi è distribuita con Licenza Creative Commons Attribuzione - Non commerciale - Non opere derivate 4.0 Internazionale. 


\title{
Poétiques du descriptif dans le roman français du XIX ${ }^{\mathrm{e}}$ siècle, A. de Georges- Métral (dir.)
}

\author{
Angela Di Benedetto
}

\section{NOTIZIA}

Poétiques du descriptif dans le roman français $d u$ XIX siècle, sous la direction d'Alice DE GEORGES-MÉTRAL, Paris, Garnier, 2015, 325 pp.

1 A partire dagli studi di Riffaterre e soprattutto di Hamon si è sviluppato lo studio della descrizione e del sistema descrittivo come uno degli argomenti principe della teoria e della storia letteraria, soprattutto dell'Ottocento. Questo volume - frutto del convegno «Poétiques du descriptif dans le roman français du xix siècle», tenutosi all'Università di Nice-Sophia-Antipolis - rappresenta una messa a punto della questione, insieme teorica e storica, e un'indagine sulle funzioni e potenzialità euristiche della descrizione. I numerosi contributi, incentrati su autori che vanno dalla generazione del 1830 sino alla fine del xIX secolo, affrontano la problematica secondo diverse prospettive critiche: quella della storia letteraria, della poetica della descrizione e della stilistica.

Bernard vouilloux (Introduction. La description et le descriptif entre l'écriture romanesque et l'histoire de l'art, pp. 19-35) introduce il volume soffermandosi sul rapporto tra la descrizione romanzesca e il discorso della storia dell'arte e illustrando le ragioni (la descrizione è per eccellenza l'approccio verbale alle opere d'arte) e le finalità (l'individuazione dei tratti distintivi della finzione letteraria rispetto ad altre tipologie discorsive) che giustificano un approccio comparativo tra i due discorsi. Partendo dai lavori di Philippe Hamon sul «sistema configurativo della descrizione» e avvalendosi della metodologia quantitativa su base digitale, Christophe sснӧсH (Intégrer ou encadrer? Les rapports changeants entre écriture descriptive et narration, de la fin des Lumières aux années 1830, pp. 39-53) compie un'analisi diacronica della poetica del descrittivo dal 
romanzo settecentesco sino a quello del primo Ottocento. L'indagine, rivolta alle modalità di integrazione dei passaggi descrittivi nel contesto narrativo e al ruolo che gli eventi diegetici assumono in tale integrazione («la motivation narrative»), mostra la principale innovazione apportata dalla pratica descrittiva di Balzac: la «motivation narrative» perde la sua funzione integrante per assumerne una distintiva. In Écrire sans décrire. L'exception idéaliste à la fin du XIX siècle (pp. 55-67), Jean-Marie SEILLAN si occupa invece di quegli scrittori (poco o affatto citati nelle storie letterarie odierne, ma che all'epoca godevano dell'appoggio del pubblico e della critica) che a fine Ottocento si schierarono contro la pratica della descrizione romanzesca: gli Idealisti. La studiosa illustra le ragioni d'ordine teologico, socio-politico ed estetico di tale rifiuto. Christophe REFFAIT (De la description comme euphémisation du savoir. La "belle fabrique de clous» de Verrières, pp. 69-80) esamina la descrizione in Stendhal, autore notoriamente refrattario a questa forma. Interessante l'analisi che mette in relazione la descrizione della fabbrica di chiodi di Monsieur de Rênal (esempio perfetto di realismo economico nel romanzo) con quella della fabbrica di spille che apre La Ricchezza delle nazioni di Adam Smith, relazione in cui Reffait coglie un intento ironico rivolto contro una delle principali nozioni economiche della società moderna: la divisione del lavoro. A partire dagli studi di Foucault sulla nascita della clinica, Bertrand MARQUER (Nosographie et poétique du descriptif. L'optique clinique, pp. 81-95) costruisce un discorso focalizzato su due questioni fondamentali: le funzioni che l'ottica clinica attribuisce alla descrizione e l'evoluzione epistemologica nel xIX secolo. Attraverso l'analisi delle opere da Balzac a Mirbeau, Marquer mostra come la descrizione si emancipi gradatamente dal realismo per assumere diverse e nuove funzioni: diegetica, allegorica, estetica.

3 Con L'Ekphrasis huysmanienne ou la description au second degré (pp. 99-114) Gaël PRIGENT apre la seconda sezione del volume, dedicata ai rapporti fra descrizione e pittura. In quanto descrizione di secondo grado, l'ekphrasis huysmaniana si sgancia, secondo il critico, dalla descrizione realista di opere d'arte vere per trasformarsi in una descrizione virtuale e fantasmagorica. La descrizione dell'opera d'arte viene allora assimilata al racconto onirico. Sempre a proposito della referenza artistica nella descrizione Arnault VAREILLE (Description et totalité. La référence muséale dans "Béatrix" de Balzac, pp. 115-129) considera il ritratto di Camille Maupin in Béatrix. Qui Balzac ricorre a una referenza museale la cui funzione, semiotica più che mimetica, è di favorire una riconfigurazione della realtà sulla base delle conoscenze e delle epifanie del lettore. Rosine GALLUZzo-DAFFLON (Référentialité et sursignifiance de la description zolienne dans "Nana", pp. 131-145) esamina tre descrizioni di luoghi in Nana osservando come la percezione e l'inventario dei luoghi e degli oggetti in esse contenute si accompagni a un'interpretazione condivisa dal lettore e dall'autore che ne decriptano il senso. La Dafflon mostra come la descrizione zoliana, costruendo una visione del mondo che eccede la rappresentazione del referente, diviene «sursignifiante».

Interessante il contributo di Pascale AURAIX-JONCHIÈRE che inaugura la terza sezione del volume dedicata alle poetiche d'autori. Il suo Poétique de la description paysagère dans "Contes d'une grand-mère" de George Sand (pp. 149-161) considera i racconti di Sand confrontandoli con la tradizione del meraviglioso cui li ascrive. Rispetto a tale tradizione, Auraix-Jonchière coglie tutti gli scarti (in primis l'ambientazione dei racconti in paesaggi familiari alla scrittrice, e non sconosciuti) che rispondono a due intenti d'autore, pedagogico e poetico. In Aux bornes du roman daudétien. Évolution de la pratique descriptive du "Petit Chose" à "Soutien de famille" à travers le motif de l'ambulation parisienne 
(pp. 163-178), Gabrielle MELISON-HIRCHWALD individua nel carattere visivo e precinematografico della scrittura di Daudet la cifra stilistica della sua opera narrativa. L'organizzazione e l'inserzione delle descrizioni infatti, piuttosto che aderire a un principio di progressione drammatica, rivelano una profonda analogia col procedimento del montaggio cinematografico. Nel suo Du poulpe à la pieuvre. Art comparé de la description chez Michelet ("La Mer"), Jules Verne ("Vingt mille lieues sous les mers") et Victor Hugo ("Les Travailleurs de la mer") (pp. 179-195), Odile GANNIER confronta i tre romanzi incentrati sullo scontro tra l'eroe e il mostro marino, soffermandosi sul rapporto tra il successo e il valore letterario. A fronte infatti dell'oblio in cui sono caduti i romanzi di Michelet e Hugo rispetto a Verne, l'autrice esalta il loro valore letterario risiedente soprattutto nell'ambizione evocativa della descrizione del polpo gigante. Anche Marie-Françoise MELMOUX-MONTAUBIN (Leurres et malheurs de la description chez Jules Verne ou l'impossible légitimation des "Voyages extraordinaires", pp. 197-209) si occupa di Jules Verne. Esaminando i Voyages extraordinaires, l'autrice mostra come la descrizione si sia costituita come un paradosso nell'elaborazione del progetto del romanziere. La ricezione dell'opera - romanzo d'avventura a carattere pedagogico e scientifico che si presta più a un'analisi tematica che estetica - ha infatti smentito le aspirazioni di Verne di essere promosso al rango di scrittore di beau style. Alain RABATEL (Donner à voir le visible? La vision opacifiante de Bloy dans "La Femme pauvre", pp. 213-224) affronta la questione dello stile di Bloy nella Femme pauvre. La descrizione deborda verso percorsi interpretativi grazie a figure tra la reticenza e la profusione ironica e sarcastica. Queste deformazioni oscurano l'ambizione di mostrare la forza del sacro nel mondo. Alexandra DelATTRE (Huysmans et la description dans "Sainte Lydwine de Schiedan". L'écrivain chrétien aux prises avec le réel, pp. 225-237) analizza le diffidenze che il mondo cattolico nutre nei confronti della descrizione considerata frutto del positivismo e al tempo stesso espressione del materialismo nel mondo moderno. Huysmans si propone nella sua opera di riconciliare la mistica e il realismo: la descrizione è il fulcro di quest'operazione. Da una parte egli esclude il compiacimento «artiste» per un linguaggio della «simplesse» tipico dei primitivi e del Medioevo; dall'altra, nell'impossibilità di attingere in prosa a questo linguaggio, adotta quello della visione. Egli instaura così una gerarchia nella rappresentazione. Il grado più basso è quello naturalista, che si riduce a una rappresentazione del reale; a un livello più alto c'è la rappresentazione dei primitivi fiamminghi che, oltre a rappresentare il reale, vi scoprono anche una verità nascosta. All'ultimo livello si pone la visione del santo che si sbarazza della mediazione del reale per arrivare direttamente alla visione di Dio. Un artista come Fra' Angelico appartiene a quest'ultima categoria. Di fatto Huysmans adotta, al posto della visione dell'artista santo, la visione dello scrittore cattolico che intende chiarire le cose del mondo. Alice de GEORGES-MÉTRAL, in Le cœur a ses ambiguités que la raison ne connaît point. Poétiques du descriptif chez Barbey d'Aurevilly, Flaubert et Hugo (pp. 239-254) si interroga sulla funzione assegnata al cuore dai tre romanzieri. Le scene descrittive, in cui l'ambiguità del cuore è messa a nudo, condensando essenzialmente due ordini di spiegazioni dei sentimenti, delle loro cause e delle modalità del loro funzionamento - fisiologico e spiritualista - formalizzano uno dei principali dibattiti del secolo. Nadia FARTAS (La simplicité de Félicité. Le simple et le confus, pp. 255-271) analizza in Un cœur simple il ricorso di Flaubert a una poetica della semplicità per rappresentare la semplicità del personaggio. Questa semplicità è un valore che si distingue da una parte dalla bêtise, dall'altra dall'agiografia. 
5 Le conclusioni del volume sono affidate a Jacques NEEFS (La prose vision de Flaubert à Proust, pp. 273-287). Il critico mostra come da Flaubert a Proust si sviluppa un'arte della prosa che produce delle descrizioni in movimento che registrano questi movimenti grazie a sonorità e ritmo. Tutti i sensi vengono dunque coinvolti trasformando la descrizione in una visione che trascende quello che comunemente si chiama realismo. 